\title{
Un interdit des maçons lyela et sa transgression (Burkina Faso)
}

\section{Luc Pecquet}

\section{(2) OpenEdition}

\section{$\checkmark$ Journals}

Édition électronique

URL : http://journals.openedition.org/span/1715

DOI : 10.4000/span.1715

ISSN : 2268-1558

Éditeur

École pratique des hautes études. Sciences humaines

\section{Édition imprimée}

Date de publication : 1 janvier 2014

Pagination : 117-145

ISBN : 9782909036434

ISSN : 0294-7080

\section{Référence électronique}

Luc Pecquet, " Un interdit des maçons lyela et sa transgression (Burkina Faso) », Systèmes de pensée en Afrique noire [En ligne], 19 | 2014, mis en ligne le 06 février 2020, consulté le 02 juin 2020. URL: http://journals.openedition.org/span/1715 ; DOI : https://doi.org/10.4000/span.1715 


\title{
Luc Pecquet
}

Un interdit des maçons lyela et sa transgression (Burkina Faso)

\section{Un interdit des maçons lyela et sa transgression (Burkina Faso)}

\author{
Luc Pecquet \\ École Nationale Supérieure \\ d'Architecture de Saint-Étienne, \\ IMAF (Institut des mondes africains)
}

« Nous ne sommes sûrs qu'il y a représentation que quand il y a comportement. »

Marcel Mauss, 1927

Chantier de construction lyela (Burkina Faso, province du Sanguié) ${ }^{1}$. Deux maçons travaillent ensemble à l'édification d'un mur circulaire d'une pièce d'habitation. Ils sont à l'extérieur du mur qu'ils montent et laissent à leurs flancs. Ils le surplombent. Au point de départ de cette nouvelle assise à édifier, ils se font face. En plaçant devant eux l'une après l'autre et les unes dans les autres les boules de terre crue et malléable, ils progressent à reculons chacun de leur côté (figures A et B).

Leurs gestes sont rapides et sûrs. Lorsqu' ils se rejoignent à l'autre extrémité de leur ouvrage, ils sont dos à dos. Pour pouvoir clore cette nouvelle assise qu'ils viennent d'ajouter au mur, ils vont devoir se retourner et à nouveau se faire face. Leur ouvrage achevé, ils le laissent. Et ils pourraient aussitôt entamer un travail similaire sur un autre mur. Mais l'un des deux maçons fait valoir à l'autre ceci : « Nous avons mis nos mains ensemble. "Ils ne peuvent reprendre leur activité avant d'avoir réglé rituellement cet impair. Et c'est ce qui va retenir notre attention.

\author{
${ }^{1}$ Agriculteurs séden- \\ taires, les Lyela, avant \\ la colonisation, sont \\ organisés en villages \\ indépendants où \\ l'habitat est dispersé. \\ Le territoire \\ villageois couvre \\ quelques kilomètres. \\ carrés et se divise en \\ quartiers, où l'habitat \\ se concentre sous forme \\ d'unités de regroupe. \\ ments des habitations, \\ ou maisons. Distantes \\ entres elles de 50 \\ à 200 mètres, ces \\ dernières sont de tailles \\ très variables (d'une \\ dizaine à plusieurs \\ centaines d'habitants) \\ Les matériaux exposés \\ ici ont été recueillis \\ entre 1988 et 1998 \\ Une mission effectuée \\ en 2013 montrait un \\ changement radical de \\ l'habitat dans la \\ dernière décennie. \\ L'habitation rectan. \\ gulaire en adobe ou \\ parpaings de ciment \\ et couverture en tôle \\ ondulée est devenue le \\ modèle courant, aussi \\ les modes de construc- \\ tion présentés ici ne \\ sont-ils plus d'actualité.
}


Figure A

Départ d'une nouvelle

assise de boules de

banco superposées.

Kyon-Esapu, mars

1989.

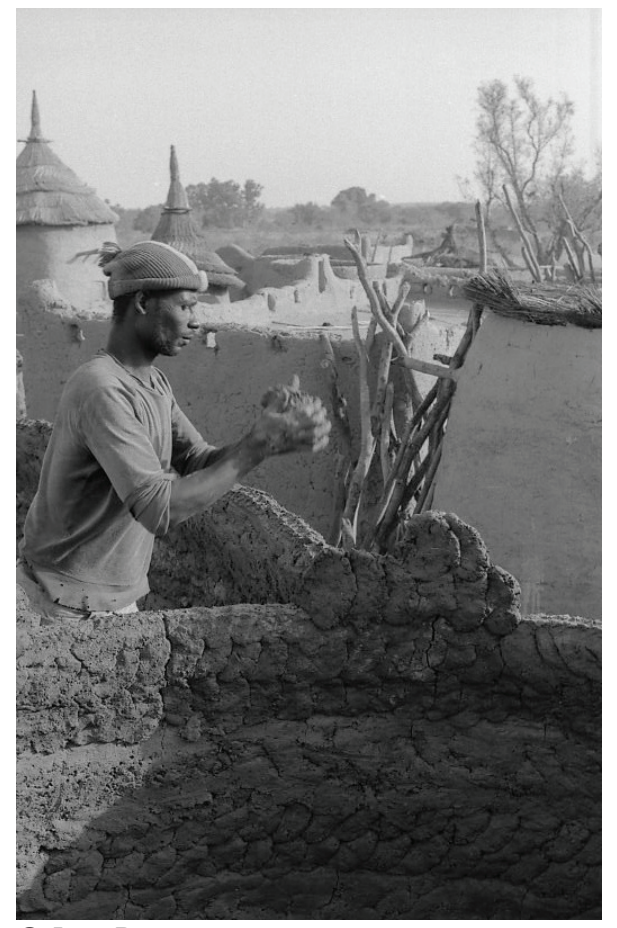

(C) Luc Pecquet

Figure B

Deux maçons au travail

sur une même assise.

Leur point de départ

est situé à peu près à

l'aplomb

de l'embrasure faite

dans le mur, là où les

boules du haut ont été

lissées. Kyon-Esapu,

mars 1989.

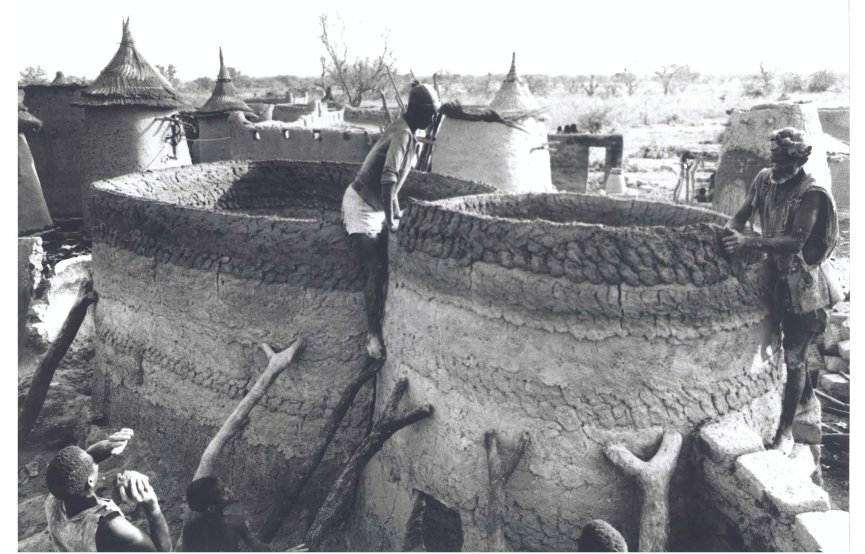

(C) Luc Pecquet 
L'interdit qu'ils auraient transgressé ? Je propose, à titre d'hypothèse, de le formuler ainsi : les mains des maçons au travail sur un même mur ne doivent pas se croiser de part et d'autre de leur ouvrage. Mais il ne m'a pas été livré comme tel. Les informations dont je dispose à ce sujet concernent une situation particulière, où l'un des deux maçons est porteur d'un anneau doté de pouvoirs. Pour se défaire des problèmes que pose à tous deux le fait d'avoir «croisé leurs mains » de part et d'autre du mur en construction, celui qui n'a pas d'anneau doit alors lui aussi se lier au fétiche qui a conféré à l'anneau sa puissance. Dans mon hypothèse, qui érige au rang des "interdits " cet acte malencontreux issu des gestes symétriques et concomitants des deux maçons, la présence de l'anneau n'est que factuelle. En d'autres termes, ce n'est pas tant le lien entre le ou les maçons et la puissance de l'anneau qu'il faudrait traiter, que leurs liens à une autre puissance incontournable des chantiers de construction : celle du matériau de construction.

La formulation de cet interdit m'est venue alors que je m'interrogeais sur une rupture et un franchissement à la fois physiques et symboliques, et posés simultanément par un rite furtif du chantier de construction d'une nouvelle maison (au sens d'ensemble d'habitations) ${ }^{2}$. Ce rite, sur lequel nous reviendrons, concerne le percement du premier mur édifié. À coups de hache, le maçon qui a la maîtrise d'œuvre perce un trou de la taille d'un poing, prélude à l'embrasure de la porte, puis il jette une poignée de poussière de terre à travers l'orifice. Cette rupture dans la continuité de la structure (tous les murs sont construits massifs), symbolisant la porte, s'accompagne de changements dans les relations entre les acteurs du rite et les structures construites. Et d'un changement de taille dans la qualité de l'espace de la maison en construction, qui d'infertile devient fécond.

Quelques précisions d'ordre général sur le chantier de construction, ses protagonistes principaux, ouvriront les propos ci-après. Ensuite, l'attention se focalisera sur les relations entre le maître maçon et son ouvrage, via la situation évoquée à l'instant : la ritualisation du percement du premier mur édifié. Ce « détour » obligé, avant d'appréhender
${ }^{2}$ On aura une descrip. tion et des données graphiques de qualité sur l'espace de la maison lyela dans Jean-Paul Bourdier et Trinh T. Minh-ha (1985: 32-49). Le principe est celui d'habitations accolées les unes aux autres, ouvrant sur une petite cour attenante à chacune d'elles et donnant elle accès à la grande cour centrale, où sont les greniers. Les Lyela sont patri. linéaires et virilocaux. Chaque habitation est le domaine d'une femme mariée (les hommes, polygames, habitent chez leurs épouses); à la tête de la maison se trouve l'aîné masculin du groupe de résidents. 
${ }^{3}$ L'essentiel des propo sitions qui suivent sur cet interdit sont issues de mon travail de thèse (Luc Pecquet,1998).

L'analyse du rite du percement du mur dans lequel elles prennent place en occupe une partie « à tiroirs » (p. 342.577), dont je ne reprends ici que quelques points.

${ }^{4}$ Voir, à ce sujet, Pecquet (1998: 578. 613) l'interdit lui-même, appelle quelques commentaires. Ce qui se dit du rite du percement du mur et, plus précisément, ce que l'on obtient comme réponses à la question de savoir pourquoi il revient au maçon de percer ce mur, permet de poser les principaux jalons des relations entre le ou les maçons et leur ouvrage. Ces dernières seront nécessairement centrales à nos propos : ce sont bien elles, en effet, que l'interdit met « en jeu ». Mais une autre dimension de ces " réponses " est à souligner : leur logique, leur rhétorique. La question de la logique ou du raisonnement à l'œuvre est, me semble-t-il, ce qui avait intéressé Michel Cartry dans les propos ci-après sur cet interdit singulier, croiser ses mains de part et d'autre d'une paroi en construction. Et c'est à ce titre que j'ai entrepris d'en reprendre ici l'hypothèse 3 .

Deux " arguments ", qui sont des leitmotivs, et que l'on doit pouvoir articuler ensemble, retiendront notre attention : si quelqu'un perce le mur à sa place, le maitre maçon quitte définitivement le site (le chantier, alors, est mort-né) ; la maison (i.e. ses murs), le maçon l'a construite d'un seul tenant, sans ouvertures : il faut la percer (faire des embrasures). Leur logique, à toute épreuve (simple, nette, économe : avare !), invite à s'interroger sur les associations d'idées dont elle serait l'expression, sur ses soubassements, sur ses arrière-plans. Elle invite également à traiter la question engagée - pourquoi ce maçon-là, seul, peut-il percer ce mur, i.e. pourquoi est-ce un rite? - en faisant usage d'une logique similaire.

Un troisième argument, laissé ici de côté, est avancé : percer ce mur et ouvrir une tombe sont des actes similaires ; qui d'autre qu'un fossoyeur est-il habilité à ouvrir une tombe? (C'est donc bien aux maçons qu'il revient de percer $\left.!^{4}\right)$. Les logiques qui " travaillent " ces arguments appuyant un même propos (le maçon perce le mur) peuvent être replacées dans ce qu'Henri Hubert et Marcel Mauss mettaient en avant à propos des raisonnements ou de la raison à l'œuvre dans la magie en soulignant, d'une part, l'importance de l'emprise des sentiments sur l'entendement et celle de leurs relations - « [les jugements de valeur 
sont "affectifs".] De même, les raisonnements se développent sur une trame de sentiments transférés, contrastés, etc., et non pas [...] suivant les lois de la contiguïté et de la ressemblance " - ; et, d'autre part, les liens très étroits unissant logique rationnelle et sentiments, qu'il n'y a pas lieu d'opposer'. C'est, globalement, dans cette veine que s'inscrivent les propos ci-après. Les notions de rupture et de continuité, de franchissements et d'attaches, de liens et de discontinuités, y tiennent une place importante. Et elles s'appliquent identiquement aux personnes et aux choses (aux structures édifiées, au matériau de construction), ces dernières paraissant s'enchâsser, s'apposer l'une à l'autre, se " jouer " sur le même mode, quand ce n'est se penser ou se poser pour équivalentes. Si ces « ingrédients " participent de ceux auxquels Michel Cartry prêtait attention, la notion d' " attache " mérite une mention particulière. Elle tient effectivement une place de premier ordre dans ses travaux sur la géomancie gourmantché - "l'homme qui sait attacher " est l'une des appellations du devin gourmantché6, et l'on ne peut omettre de renvoyer au "sacrifice d'attache" - et son importance n'est sans doute pas pour rien dans l'intérêt qu'il a un jour porté aux matériaux exposés ci-après.

Les formes que revêtent ces notions sont multiples. Y participe, par exemple, le fait que le maçon au travail, mais aussi le forgeron et la potière ne peuvent, dit-on, interrompre leur activité en cours, fut-ce en cas de décès survenant sous leurs yeux : avant de s'enquérir au sujet du défunt ou de se rendre à ses côtés, le maçon clôt la couche de mur qu'il construit, le forgeron telle étape du forgeage, et si la potière ne faisait pas de même sa poterie se briserait.

"Quand quelqu'un monte en haut (échafaudage) et on construit le banco, même si quelqu'un meurt (et est) couché à terre (i.e. meurt sous ses yeux), il va finir de construire son banco avant de descendre. Le forgeron, s'il forge (lì) ses choses, même si quelqu'un meurt (et est) couché à terre, il finit avant de venir voir. Ses choses, il faut qu'il finisse de les fabriquer (lì). [...] Si le fer est au feu, même si quelqu'un meurt, il n'y va pas. [...] Ce n'est pas qu'il forge et se lève laisser ça, pour revenir demain et recommencer à forger. Tu n'essayes pas, tu ne laisses pas. Même si la personne est morte, couchée, tu vas finir. Le banco aussi est pareil ${ }^{7}$."

Fabriquer n'est pas « une simple chose».

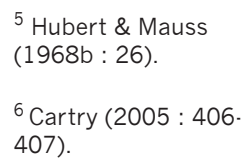

${ }^{7}$ Kyon Esapu, Beli Bayili, (18/01/1998). Ces propos sur la fabrication et sa continuité ne sont pas sans rappeler ce que soulèvent Hubert et Mauss sur la « parfaite continuité qu'il est requis d'avoir » dans l'action sacrificielle : « Les forces qui sont en action, si elles ne se dirigent pas exacte ment dans le sens prescrit, échappent au sacrifiant et au prêtre et se retournent contre eux, terribles ॥, ou bien encore, dans la note 152, « Toute faute rituelle est une coupure $[\ldots] »$ : Hubert $\&$ Mauss (1968b : 26-31). 


\section{Le travail du banco}

${ }^{8}$ Cabal propriétaire, possesseur, maître ou responsable de ; de $c \breve{r}$, commander, être maître de, posséder, et bal, homme (au féminin, cák $\tilde{\varepsilon}^{\prime} ; k \tilde{\varepsilon}$, femme). Nous ne mentionnerons plus ci-après « futur 》 maître de la maison [...], mais juste « maître de la maison » : c'est toujours ainsi qu'il apparaît dans les propos intéressants des moments où, pourtant, matériellement le kèlè n'existe pas encore.

${ }^{9}$ Lùrna, utilisé seul, désigne le maçon ou le forgeron (forger et bâtir se disent par un même terme, lù). On rencontre aussi le terme de bo lùrna, « constructeur de banco », plus concret. Ji désigne I'habitation toute entière, dont chaque pièce est également un jì. Mais ji désigne aussi le lignage (clan, segment de lignage). Ce n'est pas anodin : bâtir une nouvelle mai son, c'est escompter implanter une nouvelle lignée. Jì cák $k \tilde{\varepsilon}$, c'est la maîtresse d'habitation, mais jì cábal désigne le maître du clan ou lignage (dit aussi kwálá cábal, kwálá renvoyant au « fétiche », cǒ, du clan). II est courant d'interchanger cábal et $c a^{\prime} k \tilde{\varepsilon}$ : on peut dire $c \tilde{\varepsilon}$ cák $\bar{\varepsilon}$ pour le maître de la terre, etc. Mais dès lors qu'il s'agit du jì. cela ne désigne plus les mêmes personnes. On comprend, sous cet angle, que le premier édifié représente rituel. lement le kèlè dans son ensemble et devienne, plus tard, le lieu des autels d'ancêtres.
"Le travail du banco » (bo tum), le terme banco désignant le matériau de construction (bo, terre et eau mélangées), est l'une des expressions lyele (langue des Lyela) par lesquelles on évoque la construction des murs des habitations composant une maison. Utilisée tout autant pour des actes techniques du chantier que des aspects rituels ou sociaux, cette expression ne s'applique pas à la construction des toits en terrasse - pourtant le banco comme matière première y tient une place importante (c'est lui qui fait l'épaisseur de la toiture). Elle n'en réfère qu'à la période du chantier dont on dresse un croquis ci-après, celle où le site en travaux est placé sous l'autorité et la responsabilité d'un maçon.

Il y a trois protagonistes principaux sur un chantier de construction d'une nouvelle maison lyela : le maître d'ouvrage, le maître d'œuvre, et le matériau. Le premier est le futur " maître de la maison ", kèlè cábal, titre dont on l'affuble dès l'instant où, après de longs mois et de pénibles épreuves il obtient l'autorisation de s'installer en ce lieu qu'il convoite, et celle de creuser la terre pour en faire du banco $^{8}$. Cette double autorisation, il l'obtient du " maître de la terre " (ce cábal) au terme de rituels faits sur le site même - responsable rituel et politique, le maître de la terre est l'autorité suprême du village dans le système dit " traditionnel " (la Terre est la puissance auxquelles en réfèrent les principales règles de vie en société). Le jour même, dans une hutte végétale, il habite ce site dont il est dorénavant le maître. Quelques jours après, avec une poule dite «poule du banco ", il rend visite au second protagoniste évoqué, un maçon - ji lùrna, "constructeur d'habitation " ${ }^{9}$ - qu'il a choisi et dont il veut faire son maître d'œuvre. Le maçon ne peut que répondre positivement à la demande qui lui est faite : il prend la poule qu'on lui donne. Par ce geste, qui vaut acquiescement, il fait aussitôt de son visiteur son obligé. Il lui précise alors le jour où il doit humidifier la terre retournée pour en faire du banco, et le jour de sa venue sur le site pour débuter les travaux. En tant que maître d'œuvre, il a à charge de construire une maison viable, réunissant les conditions qui permettent d'avoir la santé, une descendance, et 
de la nourriture : un lieu où les souhaits du futur maître de la maison puissent se réaliser. Le troisième protagoniste du chantier, enfin, est le banco. Matériau réputé puissant, intelligent, susceptible de réagir aux comportements des individus, et même à leurs (mauvaises) pensées non formulées, le banco tient sa puissance de la Terre ${ }^{10}$. Pour que le chantier aboutisse, pour que la construction soit viable et, surtout, que les objectifs résumés par les souhaits évoqués à l'instant puissent se réaliser, ces trois protagonistes doivent s'entendre. Discordes, tensions et conflits que l'on se refuse à "traiter " peuvent, en effet, mener à un échec total de l'entreprise. Dans les relations que cette association propose, deux couples sont importants : celui du maçon et du maître de la maison, et celui du maçon et du banco. Le second surtout nous intéresse. Mais le situer impose de dire également quelques mots du premier. Quant à la relation entre le maître de maison et le banco, que nous laissons de côté, elle est surtout remarquable dans la période qui nous intéresse en ce que le maçon en est l'intermédiaire.

Au chantier, le maçon qui a reçu la «poule du banco » n'est plus un simple "bâtisseur " (ji lìrna), et il est plus qu'un " constructeur de banco » (bo lìrna), titre dont on affuble les autres maçons qui travaillent sur le chantier. Il est le - l'unique - bo cə́bal, le "maître du banco " : celui qui, du fait de sa relation privilégiée avec la matière première ce construction des murs, a la mainmise sur le site en chantier, le commande. Il a, dit-on, "sa main sur le kìlè " (la maison). Cette mainmise, obligée, se met en place le jour où il vient sur le site, par les actes rituels qu'il effectue. Trois actes rituels successifs l'instituent:

(1) le sacrifice du poulet que lui donne le kèlè cábal ${ }^{11}$, qui engage le maçon et la Terre (elle sait qu'il « commence le banco ", il se met sous son couvert, l'enjeu global est l'enracinement du $k \grave{\varepsilon} l \grave{\varepsilon}$ à venir $)^{12}$;

(2) le tracé du plan circulaire de la première pièce, qui appose le pouvoir du maçon sur cet espace et sur son détenteur, le kèlè cábal (il glisse son pied sur la terre, son tracé achevé « il commande la place » - cette pièce symbolise
10 « Le banco $(b o)$,
c'est de la terre $(c \tilde{\varepsilon})$ c'est de la terre $(c \tilde{\varepsilon}) \|$,
dit-on volontiers, et cela renvoie tout autant à leur matérialité qu'à leur pouvoir ou puis. sance. Mais c'est, plus précisément, de la terre et de l'eau (Pecquet, 2004 : 156-157), ce qui n'est pas indifférent au regard de l'importance de l'eau dans les « réac tions » (" paroles », en lyele, voir infra) du banco, mais aussi dans le forgeage qui est, dans les termes, assimilé à l'acte de bâtir. Que la matière soit ou puisse être pensée vivante, celà a été exposé par Mauss (1969b), mais à ma connaissance peu repris par la suite.

${ }^{11}$ Ouvrir les travaux impose de « donner un poulet à la terre » et d'avoir son assentiment, signifié par l'une des positions de l'animal sacrifié. En pays lyela, le dos du poulet jeté au sol doit toucher terre à un moment donné de ses « ébats 》 (terme que j'utilise au sens où du poulet agonisant qui fait des bonds on dit, en lyele, qu'il «se réjouit ॥), mouvements qui participent de « la présence » (M. Cartry, J.-L. Durand \& R. Koch-Piettre, 2009 : 19-25) de l'instance ; c'est, dit-on en pays lyela, le nəcílu - le grand génie de la terre - qui met le poulet sur le dos.

${ }^{12}$ Comme en français, cette métaphore (ou celle de l'implation) est associée à un rapport à la terre qui emprunte au végétal; ce qui doit «faire souche », c'est le lignage (le $j i)$. Le muret de fondation a pour 
nom jì-byòló, « maison infertile » (le rite du percement du mur inverse cette qualité : une maison viable est un espace d'engendrement), le suffixe-byòló ne s'utilisant que pour les végétaux (arbre ne donnant pas de fruits, etc.). Les rites de fonda tion kasena renvoient à des faits proches - « [i s'agit] d'implanter la future maison en un champ où elle pourra "prendre" » (Carty \& Liberski, 1990 : 134)qui sans doute se dé. clinent ailleurs $\mathrm{c}$ tiples façons. En pays batammaliba, entailler le muret de fondation frais pour y introduire une boule de terre rem. plie de graines cueillies par le maître de la terre est le premier rite de fondation. À l'idée d'an crage et de croissance (famille et maison) s'ajoute ici celle d'une nouvelle maison qui naît. (Suzanne Preston Blier, 1994 : 24-27).

13 L'habitation dont cette pièce est une com. posante sera celle de la première épouse du kèlè cəbal, et plus tard le lieu des autels d'ancêtres des maîtres de la mai. son successifs. le $k \dot{\varepsilon} l \grave{\varepsilon}$ à venir, le $\left.\operatorname{projet}^{13}\right)$;

(3) la construction de la couche de fondation de cette même pièce, construction qui assoit son emprise sur le site en tant que bo cábal (le site, peut-on dire, est dorénavant sous sa juridiction : il donnera des amendes pour toute transgression aux règles en vigueur sur le chantier).

L'emprise qu'a le maçon sur cette portion de terre, sur son "propriétaire " et sur le banco, n’est pas sans difficultés ni contreparties. Elle est, à différents égards, réciproque : le maçon est lui aussi "sous l'emprise de " autant qu' " aux prises avec » et il est, peut-on dire, pris corps et âme par ce " travail du banco ». Les actes évoqués à l'instant en posent les fondements, de la façon suivante.

Glisser son pied sur le sol pour faire le cercle de la première pièce équivaut à prendre possession des lieux, possession que rend effective semble-t-il la clôture du trait. Ce tracé, le maçon le fait sur un trait préalable, celui effectué à l'aide d'un bâtonnet passé dans un lien en U et qu'on appuie sur la terre en tournant, lien tendu. Le maçon tient verticalement en l'appuyant au sol le bâton, axe central où est enfilée l'autre extrémité du lien. Le voilà entouré par le cercle tracé ; lorsque le cercle se ferme son âme (ou son double, ywa'la') est prise. Quant au corps à corps avec le matériau, par lequel par lequel le maçon va y être étroitement "attaché » (en lyele), il s'effectue en construisant la couche de fondation. Et c'est l'achèvement de cette structure close sur elle-même, souple et figée, qui scelle ce lien puissant dont il ne pourra se défaire que rituellement, une fois son travail achevé. Pour la bâtir, le maçon projette vigoureusement, à plusieurs reprises et à pleines mains, de gros paquets de banco sur la trace de son pied. Il "terrasse " le banco, dit-on. L'image est bien celle d'une lutte : le verbe usité est celui en usage pour la lutte traditionnelle, où l'on doit plaquer au sol le dos de son adversaire. Ensuite, très rapidement, il découpe à la houe les faces internes puis externes du muret, remet ces paquets de terre humide sur les précédents, découpe à nouveau... Il obtient un muret d'un aplomb superbe, dont la tenue incertaine est compensée par la courbure du mur que vient épouser sa verticalité 
légèrement rentrée vers l'intérieur du cercle ${ }^{14}$. Sec, ce muret sera très solide. Le maçon terrasse bel et bien le banco. En contrepartie, ce dernier "l'attache " (lye $\underline{\tilde{e}}$, attacher, nouer). Aussi sa sujétion à la puissance du matériau est-elle posée comme au moment même où il le terrasse. La continuité et la compacité de ce muret sans fissures au séchage paraissent donner la mesure de l'attache qui lui est associée ${ }^{15}$.

De la mainmise qu'il a sur le site, introduite par le sacrifice évoqué puis affinée par les actes techniques et rituels qui lui succèdent, le maçon ne pourra s'en défaire qu'après l'achèvement de son travail, et par l'entremise du kèlè cábal. Après la construction des murs des habitations, il quitte le site une première fois. Il attend que le kèlè cábal l'y convie à nouveau, pour les rites et la fête de clôture. Là, à son départ définitif du site, le maître de la maison lui remet le «poulet du décrochage de la main », poulet qui fait le pendant de celui d'ouverture du chantier. À son retour chez lui, le maçon tue ce poulet. Il le tue pour se désengager, pour se détacher, pour se défaire de ses liens à la terre et au banco qu'inauguraient le premier sacrifice. Il le tue pour clore tout ce qu'il avait ouvert. Il rassemble le tout-venant qui est à terre dans la cour de sa maison, puis il égorge le volatile. Le petit tas de poussière et de déchets symbolise toutes les choses néfastes qu'il n'a pas vues ou qui se sont passées à son insu pendant le temps des travaux. Pour s'en déprendre, il fait couler dessus un peu de sang du poulet. Et par cette déprise, il retrouve quelque chose de l'ordre de son intégrité. Le voilà assuré de reprendre totalement possession de son corps (s'il ne tuait pas ce poulet il pourrait, par exemple, avoir à jamais ses mains qui tremblent), de recouvrer sa virilité (sa puissance sexuelle, ses capacités de procréation : il pourrait devenir impuissant, ou stérile), comme de ne pas perdre son savoir faire.

Pour le kèlè cábal, la mainmise du maçon sur l'espace du chantier est bénéfique, au sens où elle s'accompagne d'une protection du site. Mais elle n'est pas non plus sans difficultés : le maçon n'est-il pas venu lui ravir, «chez lui », la majeure partie de ses prérogatives? Le voilà, en effet, réduit à faire passer au second plan sa relation à la terre de ce lieu,

\footnotetext{
${ }^{14}$ Ce muret est haut de $50 \mathrm{~cm}$ et épais de $35 \mathrm{~cm}$ environ à sa base pour $12 \mathrm{~cm}$ à son sommet. La rapidité d'action et la justesse des gestes sont néces. saires à sa statique.

${ }^{15}$ Du strict point de vue technique, les particules argileuses gonflent proportionnellement à l'humidité à laquelle elles sont soumises, et elles se rétractent en séchant ; à sec elles n'occupent plus le même volume, d'où l'apparition de fissures. À moins d'être trop important, ce retrait de l'argile au séchage n'altère pas les qualités de la structure. Dans le cas présent, il est limité par la pression exercée sur le matériau en le projetant fortement au sol.
} 
16 D'autres « paroles du banco » sont évo. quées, elles apportent parfois des précisions au maçon quant au responsable de la faute. Ces « réactions » du matériau aux compor. tements transgressifs et cachés perdurent après le chantier, mais l'échelle territoriale change (on passe du quartier au village, et la terre, peut-on dire, en prend parfois le relais : elle peut choisir d'ensevelir celui qui transgresse à répétition ses interdits ( interdits de la terre », cع súsúúli) en faisant s'écrouler sur lui la toiture en terrasse de son habitation.

Le terme traduit par

« parole » est zòmè, qui désigne le langage parlé et est d'usage courant pour signifier « prob. lème ॥ (cf. François Jean Nicolas, 1953 : 559 , conversation, palabre, dispute, litige). Pour la parole donnée ou posée (promesse, souhait, etc.), le précepte, la chose dite à laquelle on en réfère, on utilise nyí, «bouche ».

${ }^{17}$ Cette responsabilité face au maçon préfigure celle qu'il aura, ensuite, face au maître de la terre, seule personne qui puisse l'expulser du village. De même, la capacité à faire

respecter les règles du chantier et à endosser la « réparation »

des transgressions

préfigurent l'exercice de

sa fonction de maître de maison. relation pourtant validée par le maître de la terre, et cela au profit d'un rapport au banco dont le maçon est le «maitre ". Il ne pourra faire, par exemple, son premier sacrifice à la terre de sa maison qu'après le départ définitif du maçon. Au quotidien, il est loin également d'avoir toutes latitudes : c'est au maçon que revient de régler toute altercation survenue sur le site, quand bien même aurait-elle eu lieu en son absence, et dès lors que cela ne relève pas des activités communes le maçon a un droit de regard sur tous les biens qui rentrent ou sortent du site en chantier, et même sur ce qu'on en prélève. Un sacrifice à faire, prescrit par un devin? L'aval du maçon est nécessaire. Il l'est aussi pour disposer des biens en circulation (produits de la chasse, de la pêche, etc.). Et il faut prévenir le maçon de tout ce qu'il s'est passé en son absence et pourrait être préjudiciable. Si le kèlè cábal s'en abstient, alors le banco s'en charge à sa place : les boules de banco avec lesquelles le maçon construit les parois lui tombent des mains, se liquéfient avant qu'il n'ait pu les assembler, ou les murs se fissurent quand ce n'est s'écroulent brutalement ${ }^{16}$. Alors le maçon cesse son travail. Il questionne, il prend connaissance des événements, puis il fixe en conséquence une amende, dont il faut s'acquitter. Son interlocuteur est le kèlè cảbal, responsable face à lui de ce qu'il se passe au kèlè, et dont le pouvoir tient surtout en sa capacité à imposer à tous le respect des règles - ou interdits, súsúlú (pl. súsúúli) - qui ont cours sur le chantier ${ }^{17}$. L'activité ne reprend qu'une fois l'amende réglée et, souvent, son produit (poulet, sorgho, etc.) préparé puis consommé.

Si le maçon ne suivait pas ce protocole, il endosserait lui-même, face au banco, la responsabilité des fautes commises par d'autres que lui, et qui normalement échoie au maître de la maison. Le maçon doit effectivement donner des amendes, en percevoir le fruit et, le cas échéant, en consommer une part. Mais s'il s'avisait de profiter de sa situation de dominant pour donner des amendes à tour de bras, ou disproportionnées, il se mettrait là encore en danger : en position, à son tour, de faire les frais d'une réaction du matériau (" parole du banco »), qui se manifesterait sur le bâti ou dans son propre corps. "Le banco est difficile " 


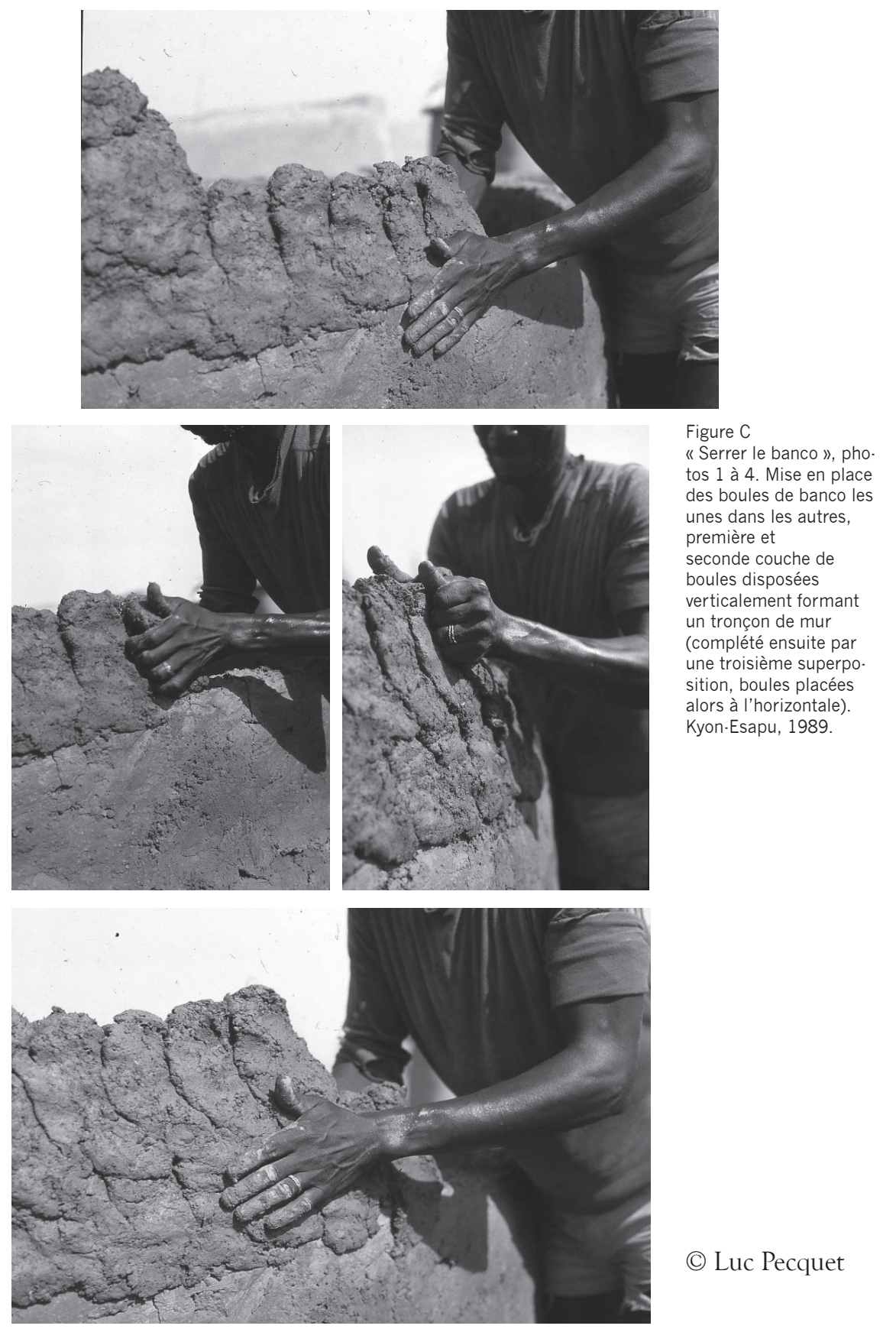


${ }^{18}$ C'est, alors, à la puissance de l'anneau qu'auraient à faire de tels maçons, et non pas à celle du maçon qu'ils souhaitent atteindre.

${ }^{19}$ Le verbe usité (kàm) est réciproque : ce que l'on comprime, ce sur quoi

I'on exerce une contrainte, en retour, en exerce une sur vous (il y a d'autres verbes pour dire serrer, comprimer, contraindre ; celui-là seul est usité pour cette expression courante). Sur cet ensemble, sur les relations entre les protagonistes du chantier, voir Pecquet, 2004.

${ }^{20}$ Aux brèves indications de la note 15 , ajoutons que chasser du matériau les vides d'air améliore ses qualités cohésives, donc l'apparition de fissures au séchage. Le comprimer fait bouger les particules argileuses qui, s'ordonnant différemment établissent entre elles des ponts, des liens (comme un chaînage), limitant alors leurs gonflements et retraits. (zum, dur, infexible, dangereux ; c'est un leitmotiv des propos sur la construction), et si la proximité du maçon au matériau le rend réceptif à ses "paroles " et lui confère du pouvoir, elle le met aussi dans une situation délicate. Son pouvoir le rend fragile, une erreur rituelle ou un comportement inadéquat est toujours possible. Le banco peut se retourner contre lui et, par surcroît, de tels écarts constituent une brèche que pourrait utiliser un maçon malintentionné travaillant sur le chantier, pour lui nuire ou jauger sa puissance. Se prémunir des agissements de tels maçons est, d'ailleurs, l'un des arguments avancés pour justifier l'acquisition d'un de ces anneaux de pouvoir évoqués précédemment ${ }^{18}$. Le maçon est dans une position dominante, mais il est continûment aux prises avec le banco. «Terrasser le banco " l'attache. Ensuite, c'est autrement qu'il entérine ses liens au matériau, en y introduisant une certaine souplesse : dorénavant, il "serre le banco ", et simultanément, comme à chacun de ses gestes, le banco l'enserre. On peut dire des contraintes qu'il fait subir au matériau, valorisées au point de " définir » son travail - « aller serrer le banco ", c'est aller travailler au chantier... -, que ce dernier réciproquement les exerce sur lui ${ }^{19}$. "Serrer le banco ", globalement, renvoie au montage des murs par assises successives de boules de banco enchâssées les unes dans les autres en les comprimant, creusant, étirant. Ces pressions à répétition que le maçon exerce sur le matériau sont importantes techniquement ${ }^{20}$. Figure 2 (photos 1 à 4 ) ci-contre.

Les interdits, ou règles du chantier sont dénommés comme indifféremment « interdits du banco » ou « interdits du maçon ». Leur transgression, globalement, porte atteinte à la réalisation de l'objectif initial : avoir une maison où sont réunies les conditions pour avoir la santé, une descendance, de la nourriture. Mais elle a deux effets distincts, que l'on peut à l'analyse associer à deux types d'interdits, et dont l'intensité varie suivant la qualité de l'infraction (volontaire, préméditée, insoupçonnée). Enfreindre les uns se manifeste dans le matériau ou le bâti - les murs, si l'on réussit à les construire, ne tiennent pas debout, s'écroulent en un éclair, dépérissent inexorablement, etc. - et touche les qualités futures de l'espace (l'efficacité de sa limite, par exemple) et des structures 
en chantier (leur solidité...). Les interdits de cette catégorie tiennent, pour l'essentiel, en celui de «bagarre ", terme couvrant des faits qui vont de la simple altercation, voire du désaccord non exprimé, à des actes violents. Enfreindre les autres interdits porte, là, directement atteinte au corps du maçon, qui peut être affecté de différents types de maux. Il n'est pas indifférent, pour notre propos, que ces secondes concernent des interventions sur les structures construites (parois, sols, et airs : mettre la toiture est interdit), que l'on va apparenter à des agressions physiques dirigées contre le maçon. Trois verbes résument ces interdictions : boucher, couvrir, percer. L'atteinte portée au corps du maçon est globalement une douleur associée à l'idée d'oppression, cette dernière renforçant l'attache initiale du maçon à son ouvrage, comme si alors ses liens au matériau se resserraient, se tendaient. Boucher les fentes dues au retrait de l'argile au séchage enferme le corps du maçon, enduire un mur "chauffe " son corps, l'étouffe. Étaler la terre latéritique dont on fait les sols est également interdit, et contribue là aussi à enfermer le maçon. Quant à aller jusqu'à damer cette terre latéritique déjà étalée pour rendre le sol plan, lisse et imperméable personne ne se risque à en décrire les éventuels effets sur le maçon.

Trois moments rituels "encadrent " le chantier. Les rites d'ouverture et de clôture du chantier sont composites, et comparativement au rite qui s'immisce entre eux, celui du percement du mur, ils se jouent sur un temps « long ». La première période rituelle, en effet, dure trois jours. Outre la mise en place des responsabilités et pouvoirs des uns et des autres, son propos essentiel tient en les différents liens ou attaches qu'elle institue. Elle débute, on l'a évoqué, par des actes rituels plutôt centrés sur le maçon et son statut de " maître du banco » (sacrifice, tracé, fondation). À cette affirmation forte du kèlè en herbe comme espace d'habitation, s'érigeant « contre " la brousse, succède un rituel qui en appelle aux futures relations entre le kèlè et la brousse $\mathrm{e}^{21}$. Au troisième jour, repli à nouveau sur le site en chantier : un repas dédié à l'assise édifiée associe tous les protagonistes du projet, pour qu'ils le réalisent. Les rites de clôture du chantier se font, eux, le jour où le kèlè cábal invite tous les
${ }^{21}$ La limite les séparant ne jouera son rôle que si conjointement elle les lie, et par de multiples jeux d'ouvertures et de fermetures (de continuités et de discontinuités), ses franchissements font l'objet de toutes les attentions. On laisse, par exemple, des espaces libres entre les habitations d'un $k$ ćlé neuf où plus tard des habitations se nicheront ; les bâtir jointives empêcherait le $k \varepsilon ́ l \varepsilon ́$ de croître (il péricliterait), et ce qui de la brousse participe de l'engendrement et le lie à l'extérieur ne pourrait y rentrer (le kélé se clôt au fur et à mesure que, par la naissance d'enfants, se tissent ses liens rituels à l'extérieur : marigots, collines, arbres...). Bien des dimensions de l'analyse de Cartry (1979) sur la brousse gourmantché comme « espace référentiel nécessaire » (ibid.: 265) à celui du village font écho à la création d'une nouvelle maison en pays lyela, où les logiques de fondation à l'œuvre sont similaires pour des territoires aussi distincts que celui de la maison, du village, du Lyòló (pays lyela ; Pecquet, 2013) : rappelons, par exemple, pour rester dans le registre précédent, l'épisode de la « fuite en brousse » de la jumelle à son mariage, condui. sant à des fermetures multiples, ou le rapport entre franchissement de la clôture et naissance gémellaire (ibid.: 277 . 282). 
22 À partir du tracé, puis avec le mur cons. truit dessus, quelque chose de la terre est enfermé et va sortir par le trou que fait le maçon au moment du rite ; sortir avec violence: le maçon doit s'en protéger (la poignée de poussière qu'il jette par le trou y pourvoit). II n'est pas pour autan quitte par rapport à la terre : le lien ouvert par le sacrifice du début, par lequel il a « la main » sur le site, il doit le clore par le sacrifice final, avec lequel « il ferme la terre $»$ et il se délie de toutes ses « attaches » au banco. participants à revenir sur le site. Ce jour-là, tous les maçons qui ont travaillé sur ce chantier se défont rituellement de liens au banco existants du seul fait d'avoir bâti : eux aussi doivent "se détacher " du banco (eux aussi, peut-on dire, le "serrent »). En d'autres termes, aux côtés de la transmission d'un kèlè viable au kèlè cəə̉bal et du « décrochage » de la main du maçon, ce sont l'ensemble des liens ou " attaches " induits par le chantier qui sont alors défaits, dénoués. De ce point de vue, il s'agit bien d'engager initialement un ensemble de liens, de relations et d'attaches, dont on se désengage ensuite lors des rites de clôture.

Par rapport à ces liens, attaches et emprises inhérentes au chantier, le rite intermédiaire du percement du mur permet au maçon de retrouver une partie de lui-même. Le petit trou qu'il perce dans le mur, en effet, libère son âme (ou son double, ywèlè) enfermée dans l'espace clôt au moment du tracé du plan. Il n'en reste pas moins " attaché " au banco, et dans un corps à corps que figure bien l'expression "serrer le banco $»^{22}$. Mais ce rite nous intéresse ici surtout en ce que les propos recueillis à son sujet, fort peu prolixes mais répétitifs, permettent d'appréhender « logiquement " les relations entre le maçon et son ouvrage, pour en venir à l'interdit qui nous intéresse. Les deux leitmotivs qui vont retenir notre attention, rappelons-le, tiennent en ces propositions, précisées ci-après : le (premier) mur, le maçon l'a construit d'un seul tenant aussi faut-il le percer (pour réaliser une embrasure...) ; c'est au maçon que revient de percer le mur, puisque si quelqu'un d'autre le fait à sa place il ne revient pas sur le site (le chantier tourne court).

\section{Une structure intacte}

La situation dans laquelle prend place le rite du percement du mur peut être résumée de la façon suivante, préalable utile aux propositions ci après, et qui en reprend plusieurs points. Les murs de toutes les pièces, de toutes les habitations en chantier, sont construits massifs. Ce sont des surfaces continues. On ne peut y ménager des ouvertures, 
des ébauches de portes, qu'une fois percé par le maçon un trou dans le premier mur (premier tracé, première couche de fondation). Avant que ne soit entamée la neuvième et dernière assise de boules de banco de cette pièce, le maître de la maison doit demander au maçon d'en percer la porte (bouche, nyí). Il lui dit de faire, à tel endroit qu'il indique (emplacement de la future porte), une petite ouverture dans ce mur qui, comme tous les autres, n'en comporte aucune. Le maçon s'exécute, dans les termes évoqués ci-dessus. Mais si le premier mur édifié est percé avant l'accomplissement du rite, et c'est peu probable, le maçon ne construit plus. Le maçon, dit-on, quitte alors le site pour ne plus y revenir. Un tel acte du kèlè cóbal se présente donc comme irréparable.

Un constat s'impose dès lors que la question du percement du mur est abordée, et c'est aussi, sur le même sujet, une référence : l'habitation, "le maçon l'a construite nâmpúm ", terme qui signifie entier, intact, vierge. L'association de ce qualificatif avec l'habitation - ji, la pièce, c'est-à-dire la première construite, qui symbolise le kèlè - exclut toute considération relative à la hauteur des murs $^{23}$. L'habitation est nâmpúm même si ses murs ne sont pas verticalement achevés. L'accent est donc mis, du point de vue formel, sur la continuité de la structure. Et percer le mur se présente d'abord comme une nécessité pratique et technique, une évidence, posée logiquement par rapport à une situation antérieure qui intéresse la maison et son constructeur. Commune, répétée (partout, par tous), cette assertion suggère que l'essentiel de l'acte du maçon réside en une modification de la situation initiale - celle qu'induit cette habitation qu'il a construite nâmpúm -, et non pas uniquement en une simple transformation de la structure. Reste qu'introduire une rupture dans l'homogénéité de la structure, ou briser ce qu'elle a d'intact, ce n'est pas simplement une nécessité pratique agrémentée d'une préséance. L'habitation construite nâmpúm par le maçon n'est pas une formule qui intéresse uniquement la structure comme objet physique : le maçon ne quitte-t-il pas le site si quelqu'un perce ce mur-là à sa place?

Les murs de toutes les maisons du chantier doivent

\author{
${ }^{23}$ Le terme usité est \\ toujours jì, pièce com. \\ me habitation dans son \\ ensemble (voir note 9). \\ On ne trouve pas, \\ pour ces propos le \\ terme de kélé (maison, \\ i.e. ici ensemble d'habi- \\ tations), mais cette \\ pièce le représente \\ effectivement, au niveau \\ des rites. Et l'on dit, \\ par ailleurs, que le kélé, \\ le maçon le construit \\ námpúm (alors même \\ qu'il laisse des espaces \\ entre les habitations, \\ qui à ce stade ne peu- \\ vent être jointives, voir \\ note 21). Enfin, le terme \\ qui signifie mur (mais \\ aussi assise, au sens \\ de couche ou portion \\ de mur), byílí, n'est pas \\ non plus usité.
}


${ }^{24}$ Aucun autre maçon n'accepterait d'en prendre la suite. Et s construire soi-même, si l'on est maçon, sa propre maison est techniquement possible rituellement cela ne l'est pas, aussi est-ce peine perdue : une telle maison n'aura pas les qualités requises. rester " intacts ". Les deux interdits (súsúúli) suivants, déjà évoqués, le montrent : percer un trou dans un mur est interdit, boucher la moindre fissure l'est également. Ils s'appliquent quels que soient la hauteur des murs et le propos de l'acte, mais la percée ritualisée du premier mur par le maçon lève en partie l'un d'eux : à sa suite, et à l'exclusion d'autres trous, on peut réaliser les passages d'une pièce à l'autre. Ces interdits semblent bien attester que la qualité des murs d'être nâmpúm, construits pleins (sans trous), est importante. Ils montrent également qu'il ne s'agit pas simplement de préserver l'intégrité de la structure. Ils ont toujours cours, en effet, après que les murs aient été percés, après l'ouverture des passages entre les pièces. Enfin, ils nous renvoient, on l'a noté, à l'investissement corporel du maçon dans son travail : leur transgression affecte corporellement le maçon. Il en va différemment avec le percement du premier mur qui, s'il intéresse effectivement les relations du maçon à son ouvrage, semble les "poser " sur un autre mode.

Percer le premier mur à la place du maçon au lieu de lui demander de le faire comme l'exige le protocole n'est pas assimilé à la transgression d'un interdit. Cet événement est d'un autre ordre. S'il se produit le maçon s'en va, et rien ne semble pouvoir le retenir sur le site. Le chantier en cours est donc irrémédiablement $\operatorname{clos}^{24}$. Il n'y a pas de réparation possible à cet acte comme s'il portait en lui, fondamentalement, une négation du travail du maçon dans sa globalité, ou de ce sur quoi son travail repose ou se fonde. Se trouve nié, dans ce qui fait la spécificité du travail du maçon (son statut de bo cábal, sa relation au banco), quelque chose d'essentiel. En d'autres termes, l'absence d'acte réparateur établit que, par-delà une étroite relation entre le maçon et son ouvrage, on est en présence d'une unité, à envisager sous forme d'une référence commune au maçon et à l'édifice. On ne voit guère que le banco, sa puissance, qui puisse prendre cette place. Percer ce mur à la place du maçon, ce serait ne pas reconnaître, nier ou ignorer, la puissance du banco, ou le banco comme puissance. Cette qualité d'être nâmpúm aurait donc comme caractéristique d'en réfé- 
rer, d'une façon particulière, au banco comme puissance. L'intact de la structure reflète l'engagement du maçon, l'entièreté de sa relation à son ouvrage ou ce qui, dans cette relation-là, est entier. Le maçon et la structure sont évidemment distincts. Mais que la construction tourne court et même s'interrompe à jamais si quelqu'un d'autre perce le premier mur les donne, peut-on dire, pour inséparables si ce n'est indistincts. Par rapport à la puissance du banco, le maçon et son ouvrage sont indifférenciés. Aussi percer ce mur à la place du maçon remet-il en cause son engagement ou, plus directement, le désengage-t-il. Il s'en va. Ce départ est irréversible (se présente tel), comme si le site, contenu dans cette maison, devenait pour toujours inapte à remplir ses fonctions.

L'habitation « intacte " n'est pas un objet ou une notion envisagée, par les Lyela, et dans les formulations, sans son constructeur. Loin de ne concerner que la structure et ses techniques de construction, l'habitation construite nâmpúm par le maçon est une expression qui nous met en présence d'une relation : le maçon et son ouvrage y sont toujours associés. Dans l'usage de cette expression, il n'y a pas une pièce bâtie d'un seul tenant qui serait totalement indépendante de celui qui l'a construite mais, toujours, une pièce édifiée telle par quelqu'un que l'on désigne, et qui doit être celui qui en perce le mur avant toute autre personne. L'expression concerne les liens particuliers établis entre le maçon et son ouvrage, et nous faisons l'hypothèse qu'elle met en valeur, entre eux, une relation de continuité. En d'autres termes, la structure édifiée et son constructeur sont tous deux investis sur ce mode du nâmpúm, les techniques utilisées pour bâtir et les représentations relatives à l'édification de l'ouvrage sont comme similaires. Pour mettre en valeur l'étroitesse des rapprochements envisageables entre l'acte technique et ses représentations, reprenons très brièvement quelques-uns des faits évoqués ${ }^{25}$.

Chacune des couches ou assises édifiées au-dessus du muret de fondation, mais aussi le mur dans son ensemble, ont pour nom byilí, et jì signifie pièce tout autant qu'habitation (ensemble de pièces). Aussi à propos de structure
25 « Tout n'est en elle [la société] que relations, même la nature matérielle des choses [...] 1), souligne Mauss (1969a : 214) au sujet des relations entre actions, pratiques collectives et représenta. tions, qui nous intéres sent ici. « II n'y a pas de représentation qui n'ait à quelque degré un retentissement sur l'action $[\ldots]$ »), insiste-t.il (ibid., p. 217), invitant dans ces quelques pages à « chercher les actes sous les représentations et les représentations sous les actes et, sous les uns et les autres, les groupes » (ibid., p. 224). Ajoutons à cette parenthèse ce propos général (ibid., p. 219) : « La notion d'efficace est commune à bien des parties de la sociologie : à la technique et à la religion en particulier », et cette notion, expurgée de la dimension religieuse qu'y associait Mauss, sera fondamentale dans le développement des travaux de Leroi. Gourhan et, à sa suite, dans le courant de la technologie culturelle où longtemps le fait religieux n'a pu être envisagé autrement qu'en termes sociaux (prendre en charge les liens entre actes techniques et actes rituels est une préoc. cupation très récente des «technologues»; voir par exemple Pierre Lemonnier, 2013). 
de la maison le vocabulaire semble soutenir, ou tout au moins permettre, un raisonnement en terme d'équivalence entre l'unité (partie) et l'ensemble. Techniquement, les contraintes physiques exercées sur le matériau par le maçon sont valorisées et recherchées. L'expression « serrer le banco ", on l'a vu, le traduit bien. C'est lors de l'édification de la couche de fondation que ces contraintes s'exercent le mieux, et l'on peut dire de l'aspect massif de cet ouvrage qu'il les traduit. Ce muret est comme d'un seul tenant, sans fissures aucunes ; c'est une construction dense, soudée, ininterrompue. Ensuite, de la mise en place de chacune des boules de banco les unes à la suite des autres par tronçons à la succession de ces tronçons puis à la superposition des couches, on est en présence d'un même souci d'homogénéité, de continuité. On retrouve ce souci, plus largement et sous de multiples formes, de la préparation du matériau - particulièrement soignée pour celui de la fondation - à l'édifice dans son ensemble. Aussi le caractère nâmpúm de la maison est-il, du point de vue technique, présent aux différents échelons du travail de construction ; le travail fait sur chacune de ses composantes y participe.

Sans le maçon, il n'y a pas d'habitation nâmpúm : il l'a construite telle. Or, le fonctionnement de l'entraide sur un chantier montre que par rapport à la réalité effective du travail, l'activité du maçon « maître du banco " (bo cəobal) peut être minime. Que d'autres, avec son accord, construisent à sa place n'enlève rien à cette qualité de l'ouvrage. Cette habitation construite nâmpúm par le maçon évoque alors, pour l'essentiel, la responsabilité du bo cábal. Certains actes ne peuvent être accomplis que par lui, il porte seul la responsabilité des rites du chantier, et il est intéressant que ce soit exprimé par des formules comme celles-ci, où la métaphore de la main rappelle, bien évidemment, son rôle technique de premier ordre ("serrer le banco ») : «Le kèlè, c'est sa main ", et celui à qui il délègue ses pouvoirs s'il ne vient pas construire doit toujours tenir compte de ce que "La main, c'est la main de l'Autre. » En jetant le banco par terre sur la trace de son pied pour construire le muret de fondation, le maçon "s'enferme dans le banco ", dit-on 

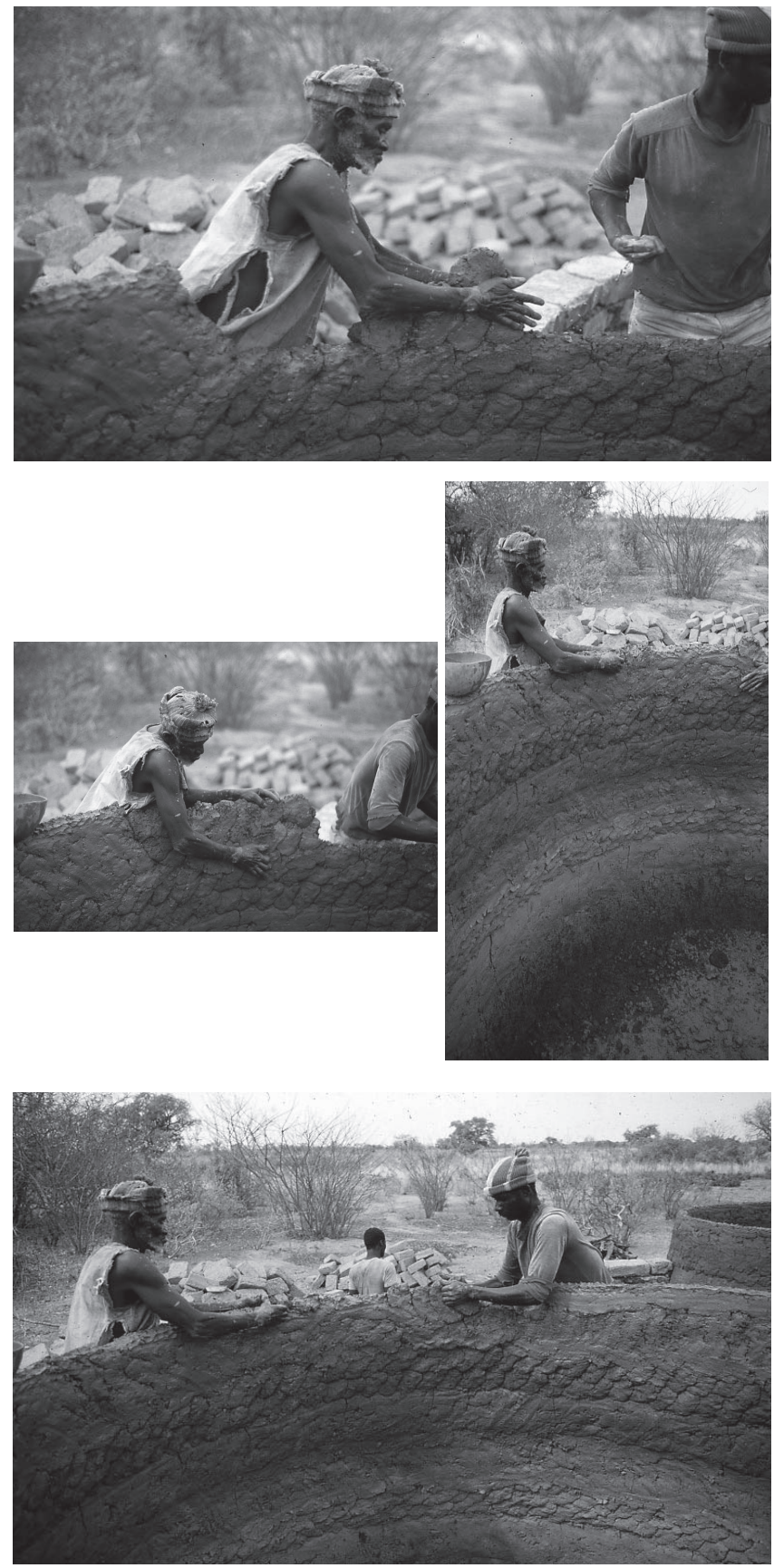

Figure D

Clore une assise. Les maçons de face : straté gie pour ne pas croiser ses mains de part et d'autre du mur.

1 Le maçon de gauche s'est retourné. II construit un tronçon de la même forme (parallélépipède, grande base en bas) que celui d'ouverture d'une nouvelle assise. Le second maçon, avec l'eau coulant de sa main, s'apprête à lisser le tronçon sur lequel il travaille.

2 Le maçon de droite ne s'est pas encore retourné ; il est affairé sur son tronçon. Celui de gauche termine le sien (jointoyer des boules). A gauche, la calebasse contient l'eau pour lisser.

3 Face à face, mais as. sez distants pour ne pas «croiser leurs mains». Le maçon de gauche, a appliqué la troisième couche de boules (horizontales) achevant sa partie, et lissé le tout.

4 Face à face, bouclage de l'assise. Gauche: finition de son tronçon (créer des cavités sur l'épaisseur, fournissant points d'accroche pour la couche suivante); Droite : mise en place de la seconde couche de boules. 
${ }^{26}$ Que le matériau de construction d'une maison puisse être " du corps », l'exem. ple des Batammaliba le pose très bien : la terre à construire est sa chair, l'eau utilisée pour l'humidifier, son sang, les cailloux qu'elle contient, ses os, et la terre à enduire, sa peau (Preston Blier, 1994 : 119). L'auteure montre bien que le bâti lui-même est traité, à différents égards, dans cette société, comme un être humain (cf. supra, note 12). La difficulté a penser la continuité de corps entre le maçon lyela et son ouvrage, que je ne peux ici que laisser de côté, n'est pas sans rappeler les propositions de Jean. Pierre Warnier (1999) revisitant la notion de culture matérielle en proposant de se pencher sur l'objet comme étant une partie intégrante du corps. L'idée d'incorporation de la dynamique des objets, souligne-t.i dans Romain Bertrand et. al. (1999) est difficile à articuler avec l'imaginaire dans lequel les objets sont pris. Outre qu'on use en architecture contempo. raine occidentale d'un langage proche (la peau d'un bâtiment et sa tex ture, etc.), la continuité de corps sollicitée ici participe, peut-on dire, de la notion d'espace -corps ouverte par Cartry (1979) parfois, assertion dont les termes n'excluent pas l'idée d'un enfermement dans l'espace circonscrit par le soubassement. Simultanément, les règles sont alors apposées sur le site (súsúúli du banco), ensemble qui atteste de son lien étroit au matériau. D'un autre point de vue, mais au même moment, la domination dont l'expression « terrasser le banco » rend compte est à l'image du résultat de l'ouvrage (son aplomb qui paraît démentir l'élasticité et l'humidité du matériau, ses qualités techniques). Elle est entière.

L'habitation construite nâmpúm par le maçon engage donc comme identiquement son constructeur et la structure qu'il édifie. Cette assertion traduit entre eux un ensemble de liens, sur le mode d'une continuité, qu'illustrent bien les transgressions d'interdits se traduisant dans le corps du maçon. L'un d'eux pose frontalement cette continuité de corps, malaisée à démêler : faire un feu contre un mur est un interdit et le transgresser, dit-on, revient à " griller le maçon " comme on le fait d'une pièce de viande ${ }^{26}$. Mais revenons à l'interdit qui nous intéresse et qui paraît attester de l'importance, mais aussi de la complexité, de ces liens " de type nâmpúm " : croiser ses mains de part et d'autre d'un mur.

\section{Croiser les mains de part et d'autre du mur}

Lorsque deux maçons travaillent à l'édification d'une même couche de banco et partent d'un même point, qu'il s'agisse d'un mur de structure continue ou discontinue, l'un attend l'édification d'un tronçon de murs (ou registre : ensemble de boules de banco assemblées, long d'environ $1 \mathrm{~m}$ et haut de $25 \mathrm{~cm}$ ) par l'autre pour engager son propre travail. Lorsqu'ils se rejoignent à la fin de la couche, ils prennent garde de ne pas se trouver à modeler ensemble un même registre, ou l'un des deux maçons s'arrête pour que l'autre fasse tout seul le tronçon de fermeture de la couche. Ces pratiques m'ont amené à formuler l'interdit évoqué, renvoyant à une situation pour laquelle les données en ma possession concernent le cas où l'un des deux maçons au travail est en relation avec une puissance, « a mangé un cem 
("médicament", remède) " : les mains des maçons au travail sur un même mur ne doivent pas se croiser de part et d'autre de leur ouvrage. Il y a de multiples façons de faire, par mégarde, se croiser les mains. Le rythme de travail est soutenu, rapide, et dans le mouvement, avoir de la retenue, marquer des temps d'arrêt ou se retirer, veiller à prendre ses distances sont autant de précautions nécessaires qui peuvent être oubliées. Le point de jonction entre les tronçons édifiés par l'un et l'autre, au début ou à la fin de la couche, est le plus délicat. Mais le début de la couche oblige, plus nettement que sa fermeture, l'un des deux maçons à attendre la pose du premier registre contre lequel il va ensuite venir appuyer le sien. C'est sa clôture qui peut plus aisément poser problème.

La transgression de cet interdit, à la différence des autres, n'impose pas l'arrêt immédiat du travail. On clôt auparavant la couche entamée. Le problème sera soulevé par l'un des deux maçons concernés une fois qu'ils sont descendus de leur échafaudage. Il entre certainement dans cette différence de traitement avec les autres interdits, qui imposent eux l'arrêt immédiat du travail, le fait que ces derniers soient commis par des tiers et non par le maçon (lequel, s'il poursuivait son travail, en endosserait les conséquences). La relation de travail ici n'est plus tripartite mais bipartite, elle intéresse spécifiquement la relation des maçons à leur ouvrage, dont on a brièvement noté ci-dessus qu'elle est soumise à des impératifs de continuité (on ne l'interrompt pas aisément, même pour des faits très importants).

Enfreindre cet interdit, pour lequel on ne cherche pas à établir qui serait à l'origine de la faute commise, se traduit physiquement : «Ton corps fera mal », dit-on. L'effet, douleur ou difficulté physique, ne paraît pas être immédiat. Tant pour l'un que pour les deux protagonistes, la transgression peut passer inaperçue. Ses conséquences, comme évoqué en ouverture de ces propos, ne m'ont été transmises que dans le cas où l'un des deux maçons est porteur d'un anneau doté de pouvoirs, acquis sur un fétiche (cǒ). L'autre maçon n'en a pas : ce doit être établi en premier lieu (on 
27 L'ingestion ne vaut que pour la substance le « médicament», mélangé au repas qui vient clore l'ensemble du processus. Dans le mode préparatoire de l'anneau il y a bien sa cuisson dans une mar. mite, par laquelle il est censé s'imprégner de ce « médicament ». peut porter un anneau sans le montrer, l'avoir dans sa poche et non à son doigt). Ensuite le problème est posé : "Nous avons mis nos mains ensemble". Alors celui qui a un anneau dit à l'autre d'amener un poulet : il lui montrera l'endroit où, comme lui, il pourra "manger le cem. ", ou " manger le cábal " (l'anneau en fer ${ }^{27}$. Pour sortir de la situation issue d'un geste malencontreux - croisement des mains de part et d'autre du mur -, les deux maçons iront ensemble en ce lieu où, après le nécessaire effectué, ils seront en relation avec la même puissance, le même fétiche (cǒ). On ne peut donc, selon ces éléments, se sortir des incidences de la transgression de cet interdit que par la création d'un lien particulier entre les deux intéressés.

$\mathrm{Au}$ " corps qui fera mal " suite à cette transgression, il n'y a pour remède que la création d'un lien, présenté comme indissoluble, entre les deux maçons. Ils deviennent tous deux, dit-on, ná-byă da-byă (pl. ; " fills de mère " " fills de père " : frères de mêmes père et mère, mais aussi « frères " au sens très large), ils " travaillent ensemble ", ils " ne doivent rien se cacher ", et c'est une situation que l'on compare à une conversion religieuse chrétienne ou musulmane en faisant valoir que l'on "rentre dans " l'une ou l'autre tout comme on doit, une fois l'interdit transgressé, " rentrer dans le fétiche ". Il est difficile de déterminer ce qui des corps de chacun (yala, corps, est un terme pluriel) serait mis à mal. Des douleurs physiques sont envisagées, par exemple dans les jambes alors que l'on est juché sur l'échafaudage en plein travail. Retenons simplement qu'à l'unité plurielle de leurs corps, qui se trouve mise à mal, les maçons n'ont d'autre solution pour pouvoir continuer à construire que celle de s'unir, de devenir solidaires, de se lier à une même puissance, voire de se donner pour "parents ". Touchés identiquement en édifiant une même structure, les maçons doivent faire passer au premier plan leur unité et comme laisser de côté ce qui les différencie. Ils créent ainsi entre eux, mais sur un autre plan, un lien comparable à celui qu'ils ont chacun, indépendamment, avec le matériau qu'ils manipulent. C'est bien par leur travail, en bouchant ou en clôturant un espace pour souder une couche du mur, un 
vide, un interstice, qu'ils se trouvent ainsi liés, et c'est pour pouvoir continuer à exercer leur métier sans être atteints dans leur corps qu'ils "mangent "le même cem. Où se situent la transgression puis sa " réparation "? Qu'est-ce qui se trouve transgressé puis ensuite "réparé »? Autrement dit, le lien à la puissance du fétiche ne vient-il pas s'ajouter à un interdit déjà présent, n'est-il pas une transposition de celui créé par le geste même (croiser les mains) qui concernerait alors, et en premier lieu, le banco ? On en revient à l'hypothèse déjà énoncée, dont il faut brièvement préciser les propositions : l'interdit s'applique aussi lorsque les maçons n'ont pas d'anneau, ou qu'ils en sont tous deux pourvus.

En l'absence d'informations sur une telle étendue de cet interdit et des actes qui seraient alors à accomplir après sa transgression, il est possible que la puissance de l'anneau (i.e. du fétiche) soit seule en jeu, qu'elle seule soit l'objet de l'interdit. Mais on le comprend mieux si l'on redonne au banco sa place primordiale. Le lien au corps mis en avant par la transgression de cet interdit n'est pas l'apanage de l'anneau, comme le montre l'exemple du feu fait contre un mur. Toutefois, l'atteinte corporelle à laquelle peut se trouver confronté le maçon est l'argument par excellence pour justifier que certains maçons "mangent le cem.». On prend un anneau, en premier lieu, "pour le mal du corps", " pour que ton corps ne fasse pas mal». Si, par exemple, un maçon pourvu d'un anneau commet une erreur et tombe de son échafaudage, il est assuré de ne rien se casser. La puissance de l'anneau lui permet de retomber sur ses pieds. Mais à cela il faut adjoindre une nuance : la chute du maçon, en relation avec l'erreur commise, peut être provoquée par le banco. Dans ces relations entre les deux puissances, où le corps est investi, retenons surtout qu'en aucun cas celle de l'anneau ne peut se substituer durablement, la santé du maçon est en jeu, à celle du banco. La force du banco « dépasse », dit-on, celle de l'anneau. Elle la supplante. Aussi peut-on envisager les répercussions corporelles de l'infraction de l'interdit qui nous intéresse comme étant, tout d'abord, issues du banco et non de l'anneau. Le banco n'est plus alors un simple intermédiaire, il passe au premier plan. 
28 De tels anneaux ne sont pas spécifiques au seul métier de maçon, mais toujours on doit spécifier devant le fétiche ce pour quoi on souhaite en acquérir un. On en « cuit » auprès des même fétiches pour d'autres activités : la lutte, la musique, ou bien encore la poterie, la vannerie.
De ce point de vue, le passage par le fétiche vient se surajouter au traitement de la transgression de l'interdit : le banco est ce qui crée initialement le lien entre les deux maçons; le lien noué parce que l'un des deux maçons porte un anneau intervient par surcroît.

L'anneau est toujours préparé pour un objectif déterminé ${ }^{28}$. Celui du maçon est de « construire le banco » avec la santé. Cet objectif doit être énoncé préalablement au sacrifice par lequel s'ouvre le procès au terme duquel on acquiert l'anneau préparé et du cem. Lorsque la transgression de l'interdit conduit un maçon à faire cette démarche, il s'inscrit dans cet objectif d'ensemble mais il évoque aussi, nécessairement, cette circonstance particulière. Le fétiche, en effet, n'accepte aucune situation trouble. Tant que subsistent des zones d'ombre, du non-dit qui le concerne, aucun des poulets ne tombe sur le dos (i.e. n'acquiesce), le procès d'acquisition de l'anneau ne peut être enclenché. Ce maçon ne vient pas uniquement pour régler l'infraction commise, ponctuelle; il veut à l'avenir pouvoir continuer à construire. Son objectif outrepasse largement le règlement de la transgression, l'évitement de ses conséquences néfastes : il renvoie au banco. L'acquisition de l'anneau n'est donc pas en contradiction avec l'hypothèse proposée, à laquelle on peut maintenant donner la tournure suivante : croiser les mains des maçons de part et d'autre du mur comporte une remise en cause du lien entre le maçon et son ouvrage, met en jeu une relation à la structure inhérente à la charge de bo cábal. L'habitation construite nâmpúm évoque, pour l'essentiel, je l'ai souligné, sa responsabilité. À l'image de celle du kèlé cábal au site, qui induit la prise en charge des erreurs commises par d'autres que lui mais dont il est le responsable, le bo cábal exerce la sienne sur tous les maçons présents. Par la transgression de cet interdit, le " maître du banco " perd en quelque sorte le contrôle de la situation, et il le perd au profit du banco.

L'objet de la "réparation " qui intervient suite à la transgression de l'interdit est le " corps qui fait mal ", image qui renvoie entre autres à une intégrité physique mise à mal. Pour ne pas être atteint dans son corps, il faut « rentrer 
dans " tel fétiche, aller "manger un cem " déterminé. C'est donc par la création de liens entre les maçons, mais aussi entre le maçon sans anneau et la puissance du fétiche, que se dénoue la situation liée à la transgression. Sous cet angle, que les maçons soient alors comme d'une même " congrégation " (je n'utilise ce terme qu'en rapport avec la comparaison à une conversion religieuse) est la conséquence et non l'objet de la "réparation ". On peut dire du lien créé entre les maçons qu'il rend effective une situation d'unité, de fusion, qui provient du geste (croiser les mains); qu'il concrétise un lien préexistant - la relation des maçons à l'ouvrage est en jeu et c'est elle qui fait l'interdit. La création sur le fétiche d'un lien particulier entre les deux maçons vise d'abord à les redonner pour distincts, et non l'inverse : par la transgression du súsúlú, le banco les lie l'un à l'autre, puis l'anneau, avec ses incidences, vient s'ajouter à ce lien. En d'autres termes, inviter l'autre maçon à aller « manger le cem » a pour fonction essentielle de redéfinir les relations au banco de chacun des maçons. Plus concrètement, ces propos se traduisent comme suit.

Pour le montage des murs, tout maçon « serre le banco ». Mais seul le bo cəóbal, par la fondation, est littéralement " attaché " au matériau, y est " enfermé ". "Serrer le banco " désigne l'ensemble des gestes inhérents à ce travail, traduit leur qualité technique, et rend compte du lien étroit unissant le maçon au matériau qu'il manipule : entre eux, l'impact est comme à double sens. Sans ce lien qui doit prévaloir sur tout autre il n'est pas de construction possible, tout comme il ne peut y avoir qu'un seul responsable du banco, le bo cə̉bal. Lorsque deux maçons qui « serrent le banco ensemble " croisent leurs mains de part et d'autre du mur, ils font prévaloir un lien de personne à personne et non plus celui de chacun au matériau ou, mais le résultat est similaire et cette option n'exclut pas la précédente, le fait qu'ils sont tous deux enfermés pareillement dans le banco. En bouchant de l'espace au même moment et au même point par le croisement de leurs mains, les maçons décalent ou rendent caducs leurs liens respectifs au matériau pour ne privilégier, involontairement, qu'une relation qui 
les concerne eux seuls ou dans laquelle leurs rôles distincts se confondent. Par ce geste les maçons créent un nouveau lien qui se substitue, ne serait-ce que l'espace d'un instant, à celui que chacun doit avoir avec le banco. En d'autres termes, boucher, souder, clore un espace à deux maçons au même instant, qu'il soit l'espace restant entre deux registres à la fin d'une couche ou que ce soient des interstices entre des boules de banco, nous met comme en présence de deux bo ciná (pl. de cábal).

Boucher équivaut à se joindre comme de façon indissoluble, à s'enfermer ensemble au même moment là où ne sont admissibles que les relations de chacun au matériau. Boucher scelle un lien et c'est ce qui fait l'interdit, le súsúlú. Pour que l'ouvrage ne perde ni son sens ni ses qualités, il ne peut y avoir partage de la charge de bo cábal, les liens qu'induisent le mur construit nâmpúm par le bo cảbal ne sauraient être divisés. On supposera donc que dénouer ce nouveau lien entre les deux maçons, hors du contexte où l'un des maçons a un anneau, est un trait essentiel des actes de "réparation " de l'interdit. Par ailleurs, aller " manger le cem. " nous met en présence d'une transposition et d'une redéfinition de ce lien par rapport à la puissance du fétiche qui est alors également impliquée, comme s'il fallait concrétiser ce lien pour faire recouvrer aux maçons leur différence. Chacun des maçons se repositionne alors dans sa relation au banco, ils la font tous deux à nouveau prévaloir : elle est nécessairement énoncée au poulet que l'on tient en main devant le fétiche avant de le donner au sacrificateur. Croiser les mains paraît bien être, hors du contexte de l'anneau, un súsúlú. Ajoutons, sans l'analyser, un dernier point pour clore ces propos.

On l'a vu, la continuité de la structure et celle du matériau créent du lien, dont attestent déjà et sous forme d'un corps à corps les expressions "terrasser " puis «serrer " le banco. Et, tant logiquement que formellement, à l'entrecroisement des mains des maçons de part et d'autre du mur comme scellant un lien entre eux répond l'image inversée de la rupture que pose le trou fait dans le mur à la place du maçon. Mais il est une autre dimension à ces questions, 
laissée en filigrane, et qui ouvre à un ensemble de propositions où les qualités de "parents » et de « corps qui fait mal " que pose la transgression de l'interdit présenté prennent un relief inattendu : celle qui consiste à reconsidérer les faits en privilégiant le sens suivant du terme nâmpúm, vierge. Précisons à ce propos, sans le commenter, qu'il y a comme un rapport charnel entre le maître maçon et son ouvrage, évoqué notamment en soulignant que si, le temps des travaux, les relations sexuelles ne lui sont pas interdites, reste que «sa virilité n'est pas grande », et qu' «il n'a plus d'eau de corps ». De ce point de vue, où la maison construite nâmpúm peut être envisagée comme vierge, l'aspect formel n'est pas vraiment en reste. Avec le trou percé dans le premier mur et la poignée de poussière que jette le maçon à travers lui, un changement de taille, on l'a noté, s'opère dans la qualité de cet espace clos qui représente le kìlè. De son affirmation en tant qu'espace inapte à générer on passe, en effet, à un kèlè fécond, à un espace de procréation. On en vient, en définitive, à un lieu où le jì, lignage et habitation (voir notes 9 et 12) pourra croître, prospérer. Cette façon d'en appeler au corps pour édifier une limite territoriale - c'est bien un propos essentiel du travail du maçon n'est pas sans rappeler (nourrir, interroger) cette notion d'espace-corps superbement posée par Michel Cartry. 


\section{Références bibliographiques}

Bertrand R., Jewsiewicki B., Warnier J.-P.

1999 "Autour d'un livre. Warnier (Jean-Pierre), Construire la culture matérielle. L'homme qui pensait avec les doigts, Paris, PUF, 1999 ", Politique africaine, $\mathrm{n}^{\circ} 76,1999 / 4$, pp. 181-195.

Bourdier J.-P., Minh-ha, T. T.

1985 African spaces. Designs for Living in Upper Volta, New-York, London, Africana Publishing Company, Holmes \& Meier Publishers.

Cartry M.

1979 «Du village à la brousse ou le retour de la question. À propos des Gourmantché du Gobnangou (Haute-Volta) », in M. Izard et P. Smith [textes réunis par], La Fonction symbolique. Essais d'anthropologie, Paris, Gallimard, pp. 265-288.

2005 "Une écriture divinatoire/Eine Orakelschrift ", in R. Baur (ed.), La Loi et ses conséquences visuelles/Das gesetz und seine visuellen folgen, Leiptzig, Lars Müller Publishers, pp. 402-429.

2009 "De la divination au sacrifice : la métaphore de l'attache ", in M. Cartry, J.-L. Durand et R. Koch-Piettre (dir.), Architecturer l'invisible. Autels, ligatures, écritures, Turnhout, Brepols (Bibliothèque de l'EPHE, Sciences religieuses ; 138), pp. 307-360.

Cartry M., Durand J.-L.\& Koch-Piettre R.

2009 "Introduction ", in M. Cartry, J.-L. Durand et R. Koch-Piettre (dir.), Architecturer l'invisible. Autels, ligatures, écritures, Turnhout, Brepols (Bibliothèque de l'EPHE, Sciences religieuses ; 138), pp. 11-38.

Cartry M. \& Liberski D.

1990 "Fondation sans fondateur (Recherches sur la notion de territoire chez les Gourmantché et les Kasena du Burkina Faso ) ", in M. Detienne (dir.) Tracés de fondation, Louvain-Paris, Peeters, pp. 85-140.

Hubert H. \& Mauss M.

1968a [1899] «Essai sur la nature et la fonction du sacrifice ", in M. Mauss, Euvres 1. Les Fonctions sociales du sacré, Paris, Éditions de Minuit (Le sens commun), pp. 193-307.

Hubert H. \& Mauss M.

1968b [1906] "Introduction à l'analyse de quelques phénomènes religieux ", in M. Mauss, Euvres 1. Les Fonctions sociales du sacré, Paris, Éditions de Minuit (Le sens commun), pp. 3-65.

Lemonnier, $\mathrm{P}$.

2013 "De l'immatériel dans le matériel... et réciproquement ! Techniques et communication non verbale ", in "La part d'“immatériel" dans la culture "matérielle" ", Journal de la société des océanistes, n 136-137, pp. 15-26. 
Mauss, M.

1969a [1927] "Divisions et proportions des divisions de la sociologie ", in M. Mauss, Euvres 3. Cohésion sociale et divisions de la sociologie, Paris, Éditions de Minuit (Le sens commun), pp. 178-268.

1969b "Conceptions qui ont précédé la notion de matière (1939) ", in M. Mauss, Euvres 2, Représentations collectives et diversité des civilisations, Paris, Éditions de Minuit (Le sens commun), pp. 161-168.

Nicolas, F. J. (RP)

1953 "Glossaire l'élé-français », Dakar, IFAN (Mémoires de l'Institut français d'Afrique noire ; 24, II), pp. 123-452.

Pecquet, L.

1998 Le Banco de l'autre. Bâtir les murs d'un ensemble d'habitations en pays lyela (Burkina Faso), thèse de doctorat Paris I-Panthéon-Sorbonne.

2004 "The mason and banco, or raw material as a power for building a Lyela home (Burkina Faso)", Paideuma 50, pp. 157-171.

2013 «Du rite à l'histoire? À propos de quelques actes et récits fondateurs lyela (Burkina Faso) ", in N. Gayibor, D. Juhé-Beaulaton et M. Gomgnimbou (dir.), L'Écriture de l'histoire en Afrique : l'oralité toujours en question, Paris, Karthala, pp. 45-69.

Preston Blier, S.

1994 [1987] The Anatomy of Architecture. Ontology and Metaphor in Batammaliba Architectural Expression, Chicago, Londres, The University of Chicago Press. 